\title{
Supporting and Understanding Socio Emotional and Sensory Based Methodologies for Individuals with Autism Spectrum Disorder through Neuroscience: A Brief Discussion
}

\author{
Eric Shyman* \\ Assistant Professor of Child Study, St. Joseph's College, USA
}

Submission:April 10, 2017; Published: June 13, 2017

*Corresponding author: Eric Shyman, Assistant Professor of Child Study, St. Joseph’s College, 155 W, Roe Blvd, Patchogue, NY 11772, USA,

Tel: 631-687-1222; Email: eshyman@sjcny.edu

\begin{abstract}
As diagnoses of Autism Spectrum Disorder (ASD) become increasingly more common among schoolchildren internationally, there also grows a pressing need to continue to evaluate multiple types of interventions that may be used with this population. In the gamut of interventional methodologies, there is vastly increasing evidence that approaches based on socioemotional and sensory-based theoretical frameworks are highly effective for individuals with ASD.

In addition to outcomes-based research, steadily growing evidence resulting from advanced neuroimaging techniques provide muchneeded biophysiological and neuroanatomical support for socioemotional and sensory-based approaches, allowing researchers and interventionists alike to better understand why certain socioemotional and sensory-based approaches are likely to be effective. The advancing neuroscientific perspective may also allow interventionists to apply more sophisticated clinical decision making skills by providing information about the likely neuroanatomical make up of individuals and the interventions that are most likely to work because of it.

Finally, though not attained yet, neuroimaging may also be able to be used to assess the effectiveness of particular interventions by allowing changes in brain function and, perhaps, even structure to be directly observed before, during, and after the intervention. This brief discussion piece will present an analysis of a sample of extant research as a means to demonstrate the emerging supporting evidence for clinical and classroom based socioemotional and sensory-based approaches using neuroscience.
\end{abstract}

\section{Introduction}

The debate regarding which methodologies should or should not be considered as part of an intervention regimen for individuals with Autism Spectrum Disorder (ASD) has been long standing and is not likely to quell any time soon. A complicated and often divisive vein of the discussion is the quality, quantity, and perspective of evidence basis used for the various approaches in question. The purpose of this article is to proffer the argument that increasing evidence from neuroscience, mainly in the form of neuroimaging, can be used to bolster legitimacy to the theoretical frameworks from which these interventions are based, as well as add to the clinical understanding of why these particular interventions are likely to work, and what variations may be likely to work for particular types of individuals. Such advances in evidence basis would enhance the legitimacy of socioemotional and sensory-based approaches as bona fide methodological frameworks of interventions for individuals with ASD.

With increased outcomes-based research attention being paid to both socioemotional and sensory-based approaches in the mainstream literature, it is ever more important to promote such findings in a cogent and organized fashion. Such careful promotion can ensure that these approaches remain a central focus not only in the research field, but also the realm of clinical and classroom practice.

Clinical and Classroom-Based Support for Socio Emotional and Sensory-Based Approaches for Individuals with ASD

Research targeting socio emotional and sensory-based interventional approaches for individuals with ASD has gained 
increasing attention in research over the past decade and a half. As a result, practitioners representing all aspects of the field have an obligation to attend to the outcomes-based research that support these types of approaches as a means to increase the likelihood that such interventions will be offered in mainstream clinical and school-based settings.

One such socio emotional intervention currently gaining significant research basis is known as the Joint Attention, Symbolic Play, Engagement, and Regulation (JASPER) protocol. A study conducted in 2013 implemented a randomized controlled trial, which compared the effects of a "treatment as usual" (TAU) protocol $(n=8)$, defined as 30 hours of ABA-based therapy per week to a structured JASPER intervention protocol $(n=7)$, implemented in place of 30 minutes of the TAU protocol per session. The participants were comprised of 15 minimally verbal children, 3-5 years old, all diagnosed with autism.

Participants were randomly assigned to either the treatment or the control group. Each group then received 3 months of baseline (in the form of TAU) and 10 weeks of intervention. Dependent variables were measured using the Structured Play Assessment (SPA), the Early Social Communication Scales (ESCS), and the Classroom Observation Measure. Results indicated that students in the treatment group showed statistically significant improvement in diversity of spontaneous play ( $\mathrm{p}<0.05$; Cohen's $d=0.81)$, less time unengaged $(p \leq 0.05$; Cohen's $d=1.63)$, and displayed more frequent requesting gestures $(\mathrm{p} \leq 0.01$; Cohen's $\mathrm{d}=1.51$ ). No significant differences were found for the ESCS variables [1].

Another well-established socio emotional method built upon relationship building and emotional connection is known as the Developmental, Individual Difference, Relationship-based Model (DIR). A well-structured randomized controlled trial conducted in 2011 investigated the effectiveness of the DIR method for preschool children. The researchers divided students into two groups:

a. A control group who received continued routine treatment without specific methodologies of DIR added.

b. A treatment group whose routine treatment was supplemented with an average of 15 hours of DIR therapy per week provided by trained parents.

The authors utilized the Functional Emotional Assessment Scale (FEAS), the Childhood Autism Rating Scale (CARS), and the Functional Emotional Developmental Questionnaires (FEDQ) to measure each dependent variable.

After incorporating the DIR therapy over three months for the intervention group, results indicated that significant gains were made on each standardized assessment for the treatment group as compared to the control group. Specifically, a 7 point difference for FEAS $(\mathrm{p}<0.05)$, a 2 point difference for CARS $(p<0.01)$, and a 7 point difference for FEDQ $(p<0.01)$ [2].
Relationship-Focused Interventions (RFI) emphasize building emotional relationships between parents/caregivers and children. One study conducted in 2005 investigated the impact of RFI on a sample of 18 dyads comprised of Korean mothers and their pre-school aged children, all of whom had disabilities. Ten dyads were assigned to an RFI group, while eight were assigned to a control group. Therapists provided training to parents via weekly group and individual intervention sessions, and mothers in the RFI group increased their use of relationshipbuilding strategies over three months.

Dependent variables were measured using the Maternal Behavior Rating Scale (MBRS), Child Behavior Rating Scale (CBRS), and Parenting Stress Index (PSI). Results indicated an overall statistically significant difference on improvement scores on the overall MBRS $(\mathrm{p}<0.001)$, as well as in the specific realms of responsiveness, affect, and achievement orientation $(p<0.001)$. There was also a statistically significant change in the affect score for the child on the CBRS $(p<0.05)$. Finally, the overall score on the PSI, as well as the parent-child relationship subdomain score was also significantly different $(\mathrm{p}<0.05$ and $\mathrm{p}<0.01$, respectively) [3].

Sensory-based approaches have also gained both attention in the literature as well as evidence-basis over the last decade. A randomized clinical trial conducted in 2013 assessed a sensory-based approach that focused on tactile, olfactory, and movement based methods. Sampling children ranging in age from 3-12 years old, participants were assigned to either an experimental treatment group, who received sensory-based therapy specifically targeting olfactory and tactile stimulation, or a control group who did not receive the additional sensorybased therapy. Nonverbal cognitive ability was assessed using the "Leiter International Performance Scale-Revised", verbal intelligence and auditory-visual-verbal association skills were measured using the "Expressive One-Word Picture Vocabulary Test". Additionally, measures of autism were assessed using the childhood autism rating scale as well as the autism diagnostic observation schedule. Results indicated that there was a significant difference in scores onstandardized cognitive assessments, with the participants in the experimental group performing higher than those in the control groupafter 6 months on all assessments $(\mathrm{p}<0.05)$ with the exception of the expressive one-word picture vocabulary test, which demonstrated an increase for both groups [4].

Researchers in 2009 conducted a randomized controlled trial investigating the effects of qigong massage therapy, a sensory based intervention derived from Chinese medicine, for 46 children between the ages of 3 and 6 . Using parent and teacher versions of the "Pervasive Pervasive Developmental Disorder Inventory" and the "Autism Behavior Checklist", results indicated that the children who received the qigong therapy displayed significant improvement in both classroom behaviors, as well as social and language skills $(p<0.01)[5]$. 
Finally, a 2010 single-subject study investigated the effects of alternative seating arrangements using a therapy ball on inseat behavior and class participation. Using six participants in either kindergarten or first grade, results indicated that for one student use of the therapy ball was highly effective, resulting in an increase in in-seat behavior and engagement, a variable though largely positive trend was evident for 4 of the children, and a negative trend was evident for one of the children. This analysis suggested that the therapy ball was more likely to be effective for children who displayed vestibular-proprioceptive seeking tendencies, while it was less effective for children who showed poorer postural stability. These results demonstrate that individual characteristics and sensory-based tendencies can have a distinct effect on clinical choices involving sensory-based interventions [6]. Other comprehensive studies examining the effectiveness of sensory-based approaches are also available in the literature $[7,8]$.

\section{Neuroscientific Evidence of an ASD Phenotype}

As demonstrated by the above sample of studies, past research evaluating the effectiveness and potential goodness of fit of interventions for individuals with ASD has depended wholly on behavioral parameters in terms of outcome measures. However, the increase in the clarity and sophistication of neuroimaging techniques as well as its contribution to deeper understanding of the potential differences in neural networks and overall brain functioning for individuals with ASD has provided an alternative, scientifically based vantage for contributing to making such clinical decisions in support of including socioemotional and sensory-based approaches. This understanding not only allows researchers to gain a deeper neurological understanding of why certain socioemotional and sensory-based interventions are effective, but can also allow interventionists an innovative way to enhance their clinical decision making protocols by using brain structures to inform what interventions are likely to work for particular individuals based on their potential neuroanatomical makeup.

Indeed, as this body of research grows, evidence suggests that individuals with ASD not only show distinct differences in brain structures and neural activation from those without ASD, but also a striking intra-group similarity. Among these similarities are regions of the brain that are associated with both sensory and emotional processing. These emerging discoveries can provide powerful evidence for supporting the inclusion of intervention methodologies that specifically target socioemotional and sensory cognitive realms, and provide ever-important evidence for understanding why and how these interventions may work.

Regarding the structure of the brain in a global context, there is clear evidence of basic structural differences between both the developing (children and adolescents) and the fully developed (adults) brains of individuals with ASD and those without. Results from a number of studies suggest multiple trends that can be found in neuroanatomical investigations of ASD. One such finding suggests that the number of Purkinje cells (cells essential in excitatory and inhibitory functions) is distinctly reduced in individuals with ASD. Additionally, brain volume appears to be $5-12 \%$ larger in young children with ASD as opposed to $1 \%$ larger in adolescents and adults with ASD. Further, the amygdala, a structure of the brain essential in emotional processing, appears to be larger in young children with ASD while reduced neuron numbers are apparent in adolescents and adults with ASD [9].

More specifically, there is mounting evidence that the sensory perceptual systems in the brains of individuals with ASD are also notably different not only in structure, but also in neural functioning and neurotransmission. For example, while it has been suggested and evidenced behaviorally that individuals with ASD have difficulty both remembering faces and distinguishing between same and different faces as well as same and different emotional facial gestures, it has been largely unknown as to the root of the perceptual difficulty. Emerging evidence from neuroscience, however, offers a host of potential explanations, citing both activation of generally unassociated "social brain network" regions in individuals with autism, as well as atypical functionality of those areas that are generally associated with successful visual discrimination [10]. Furthermore, a number of fMRI studies indicate that individuals with ASD likely have an either absentor hypoactive sensory filtration mechanism that enables individuals to bias processing toward relevant stimuli, causing the individual to give the same "neuronal attention" to all stimuli, thus causing overstimulation [11-13].

The neurological evidence extends the area of sensory processing, however, into that of emotional processing as well as emotional regulation. Because increasing understanding is being gathered about the way in which the brain likely produces and processes emotions neurologically, progress toward understanding the potential differences between individuals with ASD and those without is also taking place. Most notably, the amygdala appears to be a key factor in both emotional regulation and processing. This brain region is increasingly shown to be atypical both structurally and functionally in children, adolescents, and adults with ASD. Additionally, both the orbitofrontal-amygdala circuit of the brain, as well as the dorsolateral-prefrontal hippocampus is related to such difficulty in processing and regulating emotion. These areas have also been shown to be structurally and functionally different in individuals with ASD [14].

Further support of the role of the amygdala in the phenotypic outplay of individuals with ASD is multitudinous in the literature [15-17].

\section{Connecting the Emerging Phenotype with ASD Intervention}

The above information establishes a clear intergroup difference as well as an emerging intragroup consistency between 
the neurological makeup of individuals with and without ASD. Though the information is largely framed in a neuroscience context, its implications are directly related to education and intervention.

While some might question the relevance of neuroscientific findings for teachers and other non-medical clinicians or interventionists, it is important to note the increasing direct connection between interventional efficiency and neural anatomy and functioning, and the effect that advances in neuroscience appear to have on classroom-based interventions $[18,19]$. Additionally, the number of journals dedicated to connecting the study of neuroscience and education directly has increased to include titles such as Mind, Brain, and Education (Wiley), Trends in Neuroscience and Education (Elsevier), and Educational Neuroscience (Sage). Clearly, there is not only an increasing interest, but an increasing presence of legitimate scientific research to necessitate such journals. This increased interest applies directly to individuals with ASD.

Perhaps the most promising element of connecting neuroscience with interventional practices is that of brain plasticity. Brain plasticity refers tothe ability of the brain to alter its structural and functional nature in response to a variation in environmental demands, which could include training and specific intervention [20]. It is through brain plasticity that interventions that are tailored to likely neuroanatomical and neuro-functional conditions and functions are likely to work.In this vein, there are a number of studies suggesting that deepening social interactions, especially those rich in emotional exchanges, can have a direct influence on neurological development in the brain areas corresponding to language, emotion, and social interaction likely facilitated through the process of brain plasticity. Specifically, Dawson cites the promise of interventions that target parent-child interactions [21].

Further, extending the notion of using neuroscience evidence simply as an informer of intervention, but also as a potential means of evaluation is an important potential for this frame of inquiry. That is, with increasing sophistication and availability to educational and neuroscience researchers, formative assessment of neurological functioning can actually serve to evidence the effectiveness of particular interventions in addition to behavioral observations, strengthening theessentially subjective nature of pure behavioral observation [21].

While the above report handles only a small sampling of current findings, the literature continues to publish studies that evidence the effectiveness of socioemotional and sensory-based approaches for individuals with ASD.

\section{Implications and Future Directions}

Because the area of neuroscience and education is relatively new and research directly connecting intervention with neuronal changes does not yet exist in any significant or practically available way, there are many implications and future directions for this realm of inquiry. First, researchers in the areas of education and neuroscience must forge closer relationships in order to pool their expertise and determine valid inquiry methods that facilitate connections between brain imaging and intervention research.

Second, the field of ASD as a whole must expandits willingness toinclude interventions that are often seen as "alternative" into better designed studies that involve neuroimaging to earnestly evaluate these approaches. That is, while much research focuses on behaviorally-based interventions for ASD, more clinic and school-based programs must be willing to be open to implementing socioemotional and sensory-based approaches, especially considering the emerging neuroscientific evidence.

Third, but certainly not finally, more mainstream journals, especially those directly handling research involving ASD and other developmental disorders, must open to seminal research connecting neuroscience and interventions in ASD, including theoretical explorations, that explore this area of inquiry in order to encourage and grow promising research endeavors.

\section{Reference}

1. Goods KS, Ishijima E, Chang Y, Kasari C (2013) Preschool based JASPER intervention in minimally verbal children with autism: Pilot RCT. J Autism Dev Disord 43(5): 1050-1056.

2. Pajareya K, Nopmaneejumruslers K (2011) A pilot randomized controlled trial of DIR/Floortime ${ }^{\mathrm{TM}}$ parent training intervention for preschool children with autism spectrum disorders. Autism 15(2): 1-15 Birth Issues in Perinatal Care.

3. Kim JM, Mahoney G (2005) The effects of relationship focused intervention on Korean parents with their young children with disabilities. Research in Developmental Disabilities 26: 117-130.

4. Woo CC, Leon M (2013) Environmental enrichment as an effective treatment for autism: A randomized controlled trial. Behav Neurosci 127(4): 1-11.

5. Silva LM, Schalock M, Ayres R, Bunse C, Budden S (2009) Qigong massage treatment for sensory and self-regulation problems in children with autism: A randomized controlled trial. Am J Occup Ther 63(4): 423-432.

6. Bagatell N, Mirigliani G, Patterson C, Reyes Y, Test L (2010) Effectiveness of therapy ball chairs on classroom participation in children with autism spectrum disorders. Am J Occup Ther 64(6): 895-903.

7. Pfeiffer BA, Koenig K, Kinnealey M, Sheppard M, Henderson L (2011) Effectiveness of sensory integration interventions in children with Autism Spectrum Disorders: A pilot study. Am J Occup Ther 65(1): 7685.

8. Case-Smith J, Weaver LL, Fristad MA (2015) A systematic review of sensory processing interventions for children with Autism Spectrum Disorders. Autism 19(2): 133-148.

9. Courchesne E, Pierce K, Schumann CM, Redcay E, Buckwalter JA, et al. (2007) Mapping early brain development in autism. Neuron 56(2): 399-413.

10. Behrmann M, Cibu T, Humphreys K (2006) Seeing it differently: Visual processing in autism. Trends Cogn Sci 10(6): 258-264. 
11. Baker AE, Lane A, Angley MT, Young RL (2007) The relationship between sensory processing patterns and behavioural responsiveness in Autistic Disorder: A pilot study. J Autism Dev Disord 38(5): 867-875.

12. Belmonte MK, Cook EH, Anderson GM, Rubenstein JL, Greenough WT, et al. (2004) Autism as a disorder of neural information processing: Directions for research and targets for therapy. Mol Psychiatry 9(7): 646-663.

13. Ben-Sasson A, Hen L, Fluss R, Cermak SA, Engel-Yeger B, et al. (2009) A meta-analysis of sensory modulation symptoms in individuals with Autism Spectrum Disorders. J Autism Dev Disord 39(1): 1-11.

14. Bachevalier J, Loveland K (2006) The orbitofrontal-amygdala circuit and self-regulation of social-emotional behavior in autism. Neurosci Biobehav Rev 30(1): 97-117.

15. Baron-Cohen S, Ring HA, Bullmore ET, Wheelwright S, Ashwin C, et al. (2000). The amygdala theory of autism. Neurosci Biobehav Rev 24(3): 355-364.

16. Mosconi MW, Hazlett HC, Poe MD, Gerig G, Smith RG, et al. (2009) Longitudinal study of amygdala volume and joint attention in 2- to4-year old children with autism. Arch Gen Psychiatry 66(5): 509-516.
17. Schultz RT (2005) Developmental deficits in social perception in autism: The role of the amygdala and fusiform face area. Int J Dev Neurosci 23(2-3): 125-141.

18. Hardiman M, Rinne L, Gregory E, Yarmolinskaya J (2012) Neuroethics, neuroeducation, and classroom teaching: Where the brain sciences meet pedagogy. Neuroethics 5(2): 135-143.

19. Nouri A, Mehrmohammadi M (2012) Defining the boundaries of neuro education as a field of study. Educational Research Journal 27(1,2): $1-25$.

20. Draganski B, Gaser C, Busch V, Schuierer G, Boghdan U, et al. (2004) Neuroplasticity: Changes in grey matter induced by training. Nature 427(6972): 311-312.

21. Dawson G (2008) Early behavioral intervention, brain plasticity, and the prevention of Autism Spectrum Disorder. Dev Psychopathol 20(3): 775-803.

\begin{tabular}{l} 
Your next submission with Juniper Publishers \\
will reach you the below assets \\
- Quality Editorial service \\
- Swift Peer Review \\
- Reprints availability \\
- E-prints Service \\
- Manuscript Podcast for convenient understanding \\
- Global attainment for your research \\
- Manuscript accessibility in different formats \\
( Pdf, E-pub, Full Text, Audio) \\
- Unceasing customer service \\
Track the below URL for one-step submission \\
https://juniperpublishers.com/online-submission.php \\
\hline
\end{tabular}

